\title{
APLICACIÓN DEL SISTEMA DE EVALUACIÓN FORMATIVA Y COMPARTIDA A UN PROYECTO GAMIFICADO
}

Application of a formative and shared assessment system to a gamified project

Aplicação do sistema de avaliação formativa e partilhada a um projeto gamificado

\author{
Manuel Cañas Encinas (1) \\ Noelia García Martín (2) \\ Ruth Pinedo González (3) \\ César Caballero San José (4)
}

(1) Universidad de Valladolid, España. Teléfono: +34 34 921112294. Correo electrónico: manubernardos97@,gmail.com

(2) Universidad de Valladolid, España. Teléfono: +34 34 921112294. Correo electrónico: noeliagarcia125@gmail.com

(3) Universidad de Valladolid, España. Teléfono: +34 34 921112294. Correo electrónico: ruth.pinedo@psi.uva.es

(4) Universidad de Valladolid, España. Teléfono: +34 34921112294

\section{Resumen}

En este artículo se expone cómo se ha introducido la Evaluación Formativa y Compartida en La misión de la luz negra, un proyecto de aprendizaje expresivo en Educación Física que se ha aplicado durante el curso académico 2018/2019 en un aula de Educación Primaria de un centro rural de Segovia. Uno de los elementos fundamentales de la gamificación educativa es la retroalimentación constante gracias a la cual el alumnado puede conseguir niveles, puntos o badges. Dicha retroalimentación se ha aprovechado para desarrollar y aplicar diferentes instrumentos de Evaluación Formativa y Compartida, tales como el Informe de la misión semanal que recogía una evaluación cualitativa y cuantitativa tanto grupal como individual. Tras finalizar el proyecto, el alumnado indicó que este sistema de evaluación les permitió autorregular su aprendizaje, reconociendo así lo que debían continuar realizando o mejorar.

Palabras clave: Evaluación formativa y compartida; proyecto expresivo; gamificación; Educación Primaria; Educación Física 


\begin{abstract}
This article explains how formative and shared assessment has been introduced in The mission of the black light, an expressive learning project in physical education that has been applied during the 2018/2019 academic year in a Primary Education classroom in a rural centre in Segovia. One of the fundamental elements of the educational gamification is the constant feedback thanks to which the students can obtain levels, points or badges. This feedback has been used to develop and apply different instruments of formative and shared evaluation, such as the Weekly report of the mission that collected a qualitative and quantitative evaluation both group and individual. At the end of the project, students indicated that this assessment system allowed them to self-regulate their learning, thus recognising what they should continue to do or improve.
\end{abstract}

Keywords: Formative and shared assessment; expressive project; gamification; Primary Education; Physical Education

\title{
Resumo
}

Este artigo explica como a Avaliação Formativa e partilhada foi introduzida em La misión de la luz negra, um projeto de aprendizagem expressivo realizado em Educação Física durante o ano letivo 2018/2019 numa aula do Ensino Primário num centro rural de Segóvia. Um dos elementos fundamentais da gamificação educativa é o feedback constante, através do qual, os alunos podem conseguir obter níveis, pontos ou crachás. Este feedback serviu para desenvolver e aplicar diferentes instrumentos de Avaliação Formativa e Partilhada, tais como o Relatório da missão semanal que recolhia uma avaliação qualitativa e quantitativa, tanto em grupo como individual. No final do projeto, os alunos indicaram que este sistema de avaliação lhes permitiu auto-regular a sua aprendizagem, identificando assim o que deviam continuar a fazer ou a melhorar. Palavras-chave: Avaliação formativa e partilhada; projeto expressivo; gamificação; Educação Primária; Educação Física 


\section{Introducción}

La gamificación educativa es una metodología que "utiliza mecánicas, dinámicas y elementos propios de los juegos de manera independiente pero sistemática, sin que tengan que conformar en sí mismos un juego" (Sebastiani \& Campos-Ruis, 2019, p.22). Entre otros beneficios, Borrás (2015) expone que la gamificación permite la retroalimentación constante, así como resultados más medibles (niveles, puntos y badges). Precisamente por ello, a lo largo del proyecto expresivo que se propone se ha aplicado la evaluación formativa y compartida como eje de la gamificación. De esta manera, las retroalimentaciones no solo han permitido establecer la consecución o no de los puntos o niveles, sino que se han promovido el aprendizaje del alumnado y la mejora de la actividad docente (Pérez-Pueyo \& López-Pastor, 2017).

Esta experiencia gamificada titulada La misión de la luz negra ha tenido como avatares a los personajes de Los increíbles. La narrativa se ha basado en que el alumnado grupalmente ha tenido que enfrentarse a la misión de transportar a los espectadores a la dimensión de la luz negra, para lo cual ha sido necesaria una progresiva formación en Teatro Negro. Se establecieron cinco estatus, cada uno de ellos representados por un nivel. Gracias a la acumulación de bonus de energía (puntos), cada grupo pudo ascender progresivamente de nivel en la llamada La pila del poder grupal. En este proceso tuvieron gran importancia los diferentes instrumentos diseñados y aplicados de evaluación formativa y compartida.

\section{Contextualización}

Esta experiencia se ha desarrollado en un aula de un centro rural agrupado de Segovia durante el curso académico 2018/2019. El proyecto de aprendizaje expresivo gamificado se ha aplicado con 15 participantes de $3^{\circ}$ y $4^{\circ}$ de Educación Primaria en el área de Educación Física.

\section{Diseño y desarrollo}

La Tabla 1 muestra las técnicas e instrumentos de evaluación formativa y compartida utilizados para evaluar los aprendizajes. Las anotaciones del Diario de campo se entregaban al alumnado en un Informe de la misión semanal que recogía una evaluación cualitativa y cuantitativa tanto grupal como individual (Figuras 12 ). Se indican las iniciales de los niños para distinguir el feedback que se da a cada miembro del grupo. 
Tabla 1.

Técnicas e instrumentos del proyecto La misión de la luz negra

\begin{tabular}{ll}
\hline \multicolumn{1}{c}{ Técnicas } & \multicolumn{1}{c}{ Instrumentos } \\
\hline Observación sistemática & Diario de campo \\
& Rúbrica de evaluación de la representación grupal \\
\hline Coevaluación & Rúbrica de evaluación de la representación grupal \\
\hline Demanda de productos & Lista de control para evaluar el Cuaderno de la misión \\
\hline Autoevaluación & Escala gráfica individual (diana) \\
& Escala gráfica grupal (diana) \\
& Rúbrica de evaluación de la representación grupal \\
& Escala de autocalificación numérica \\
\hline
\end{tabular}

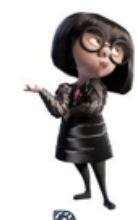

踏

Has comenzado la misión con una alta implicación. Además. eres capaz de motivar al resto de personas del equipo. En la rutina veo/pienso/me pregunto te planteaste el funcionamiento de las luces, una pregunta buenísima. Hubiese sido necesario haber pensado un mayor número de preguntas.

\section{㩆}

Tienes que participar más en las sesiones. Cumples con las normas, pero te tienes que implicar más.

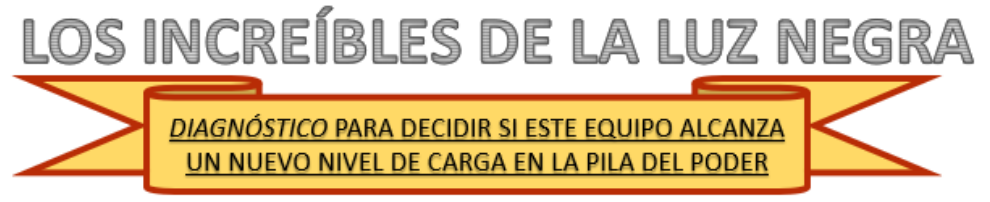

(3)

Buena participación en las rutinas y buena actitud en cada una de las sesiones, cada día te comportas mejor.

睓

Parece que te está gustando la expresión corporal. Te involucras en la sesión, pero tienes que aprender a ser menos impaciente. La rutina veo/pienso/me pregunto es bastante buena, hubiese estado perfecta si hubieras escrito un mayor número de preguntas.

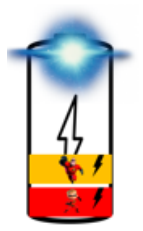

\section{西}

Participas activamente en la sesión y motivas y respeta a los compañeros y compañeras. La rutina de pensamiento veo/pienso/me pregunto es realmente buena

Figura 1. Modelo de Informe de diagnóstico semanal (anverso)

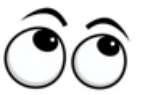

Enhorabuena, habơ

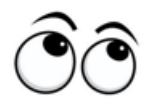

Toda esta energía significa que, en general, habéis participado bastante en la sesión y habéis funcionado como grupo. De manera general, quiero indicaros que os ha faltado escribir el porqué de vuestra conclusión en la rutina Titular (reto 3). Es importante que en las siguientes rutinas justifiquéis la respuesta con vuestras ideas. También os falta participar en las asambleas que se realizan.

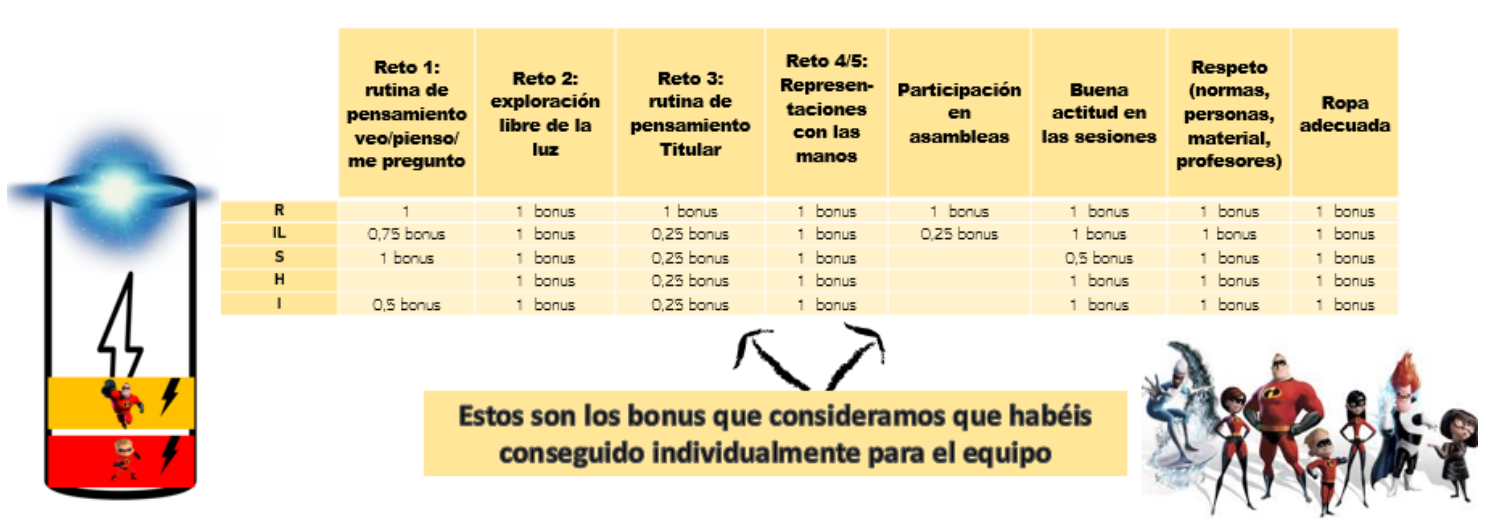

Figura 2. Modelo de Informe de diagnóstico semanal (reverso) 
El proyecto duró cuatro semanas, por lo que cada grupo recibió cuatro Informes de la misión. Estos se adaptaban a las tareas/actividades realizadas a lo largo de cada semana, siendo constantes los criterios recogidos en la Figura 3. Además, se tenían presentes las autoevaluaciones de cada sesión y el resto de los instrumentos para otorgar los correspondientes bonus de energía a las personas de cada uno de los grupos.

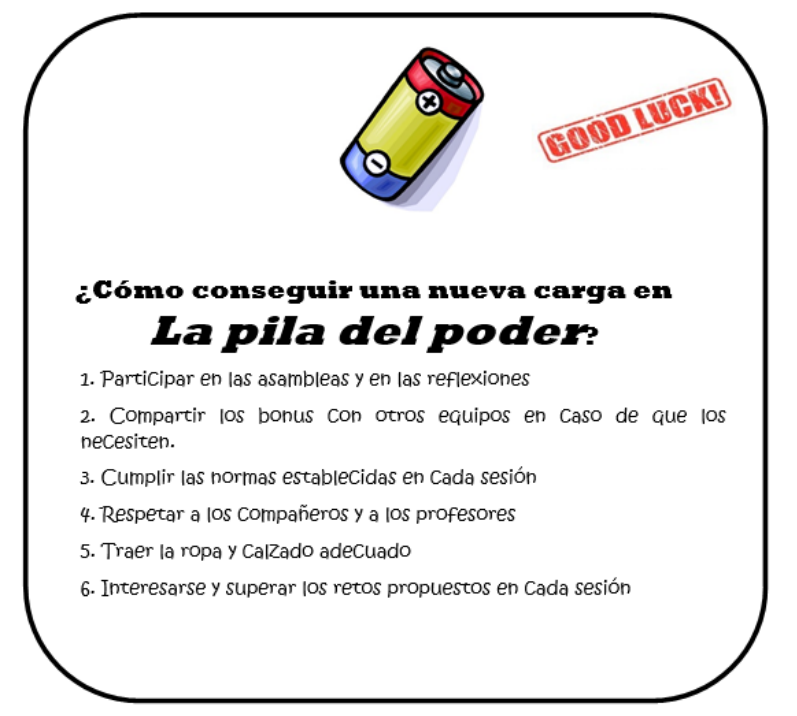

Figura 3. Criterios para alcanzar un nuevo nivel de carga en La pila del poder

Por otra parte, para asegurar que el alumnado revisaba la evaluación facilitada y no únicamente si se había alcanzado un nuevo nivel, cada persona del grupo tuvo que firmar en una hoja elaborada para tal fin después de haber revisado dicha evaluación (Figura 4) e indicar verbalmente al docente qué era aquello que tenía que continuar realizando o mejorar. Tras esto, cada grupo pudo canjear los bonus de energía por el nivel correspondiente (Figura 5).

Por otra parte, al haber asumido un modelo de evaluación compartida, el docente ha facilitado al alumnado, además de los informes, todos aquellos instrumentos con los que se evaluaban sus producciones (rúbricas, ensayos, representaciones, etc.).

En la última sesión, tras la autocalificación del alumno o alumna mediante la escala de autocalificación, el profesor indicó en ella su propia opinión sobre cada indicador evaluado. Si existían diferencias importantes entre ambos, el profesor anotaba su opinión sobre cada indicador en dicha escala y se reunía con el alumno o la alumna para dialogar la calificación alcanzada en el proyecto. Uno de los indicadores que se evaluaban era si se habían tenido presentes las evaluaciones de los informes (Figura 6, en Anexo). 


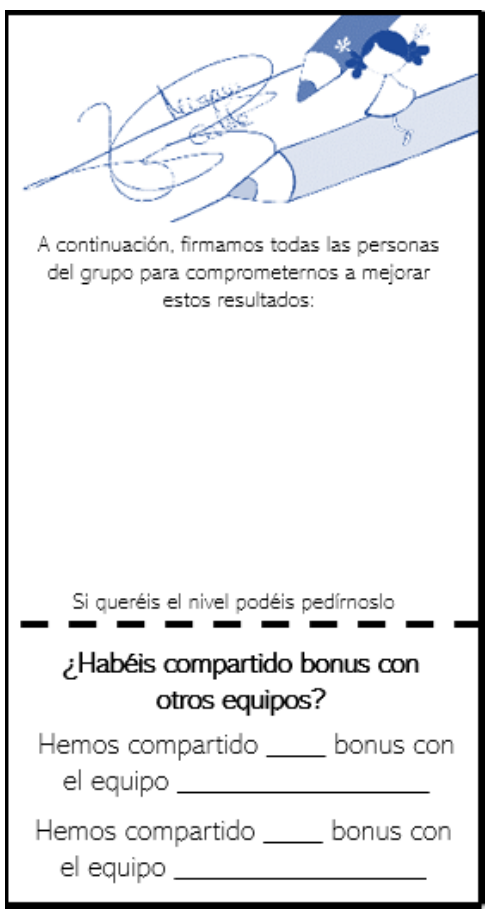

Figura 4. Ficha de compromiso grupal para mejorar el resultado de la evaluación
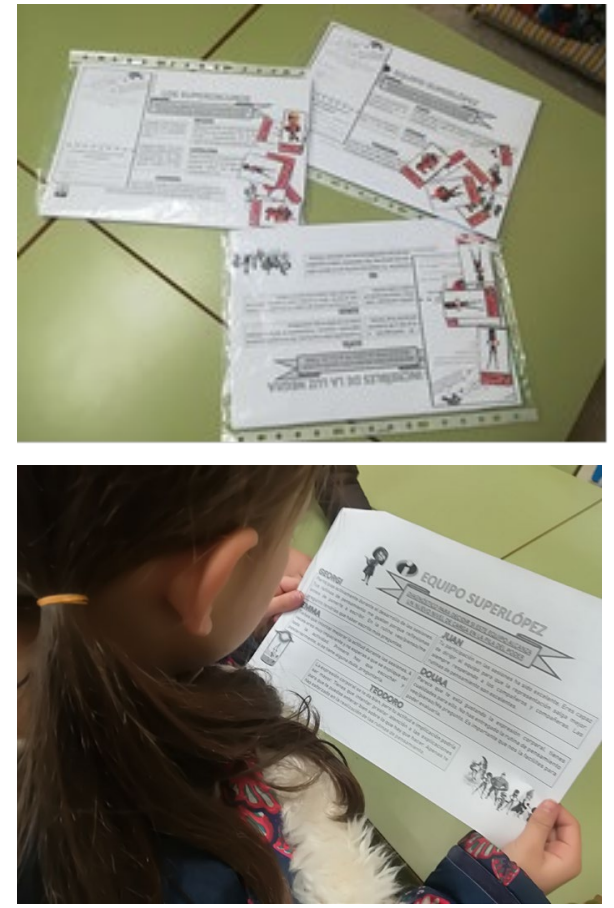

Figura 5. Revisión de las evaluaciones en los Informes de la misión

\section{Evaluación y conclusiones}

Esta experiencia ha permitido comprobar los beneficios de la aplicación de la evaluación formativa y compartida en el aula. Al finalizar la última sesión, se facilitó a cada alumna o alumno un cuestionario de evaluación del proyecto. Una de las preguntas era valorar el modelo de evaluación que se había seguido a lo largo de la unidad. Sus respuestas indican claramente valoraciones positivas sobre el modelo de evaluación seguido. Algunos ejemplos son:

- "Sí, porque ahora he mejorado"

- "Sí, para saber lo que hacía bien”

- "Sí, porque con la evaluación hay errores y también porque se pueden mejorar"

- "Sí, porque también digo cómo lo he hecho"

Aunque el alumnado no estaba acostumbrado a este modelo de evaluación, progresivamente hemos podido comprobar que les permitía autorregular su aprendizaje, así como establecer interacciones grupales gracias a la realización de autoevaluaciones y coevaluaciones. Además, la mayoría de las personas de los grupos aprovecharon las retroalimentaciones proporcionadas para mejorar en determinados aspectos e intentar conseguir un mayor número de bonus. 


\section{Referencias}

Borras, O. (2015). Fundamentos de la gamificación. Universidad Politécnica de Madrid.

Pérez-Pueyo, A., \& López-Pastor, V. (2017). Evaluación formativa y compartida: experiencias de éxito en todas las etapas educativas. Universidad de León.

Sebastiani, E., \& Campos-Rius, J. (2019). Gamificación en Educación Física. Reflexiones y propuestas para sorprender a tu alumnado. Barcelona: INDE. 


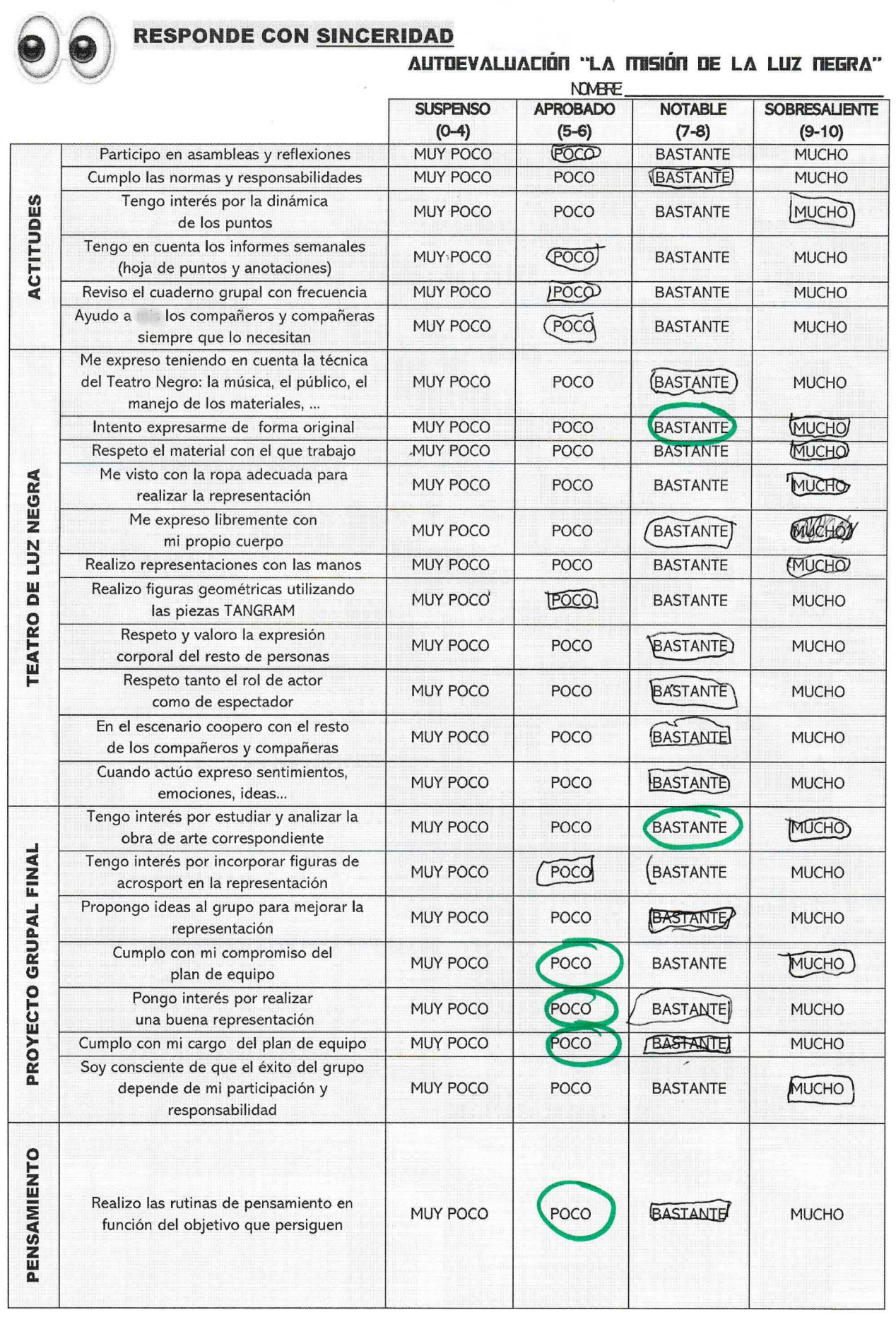

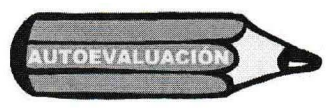

Figura 6. Ficha de autocalificación del proyecto expresivo 